\title{
Strip Detector for the ATLAS Detector Upgrade
}

FOR THE High-Luminosity LHC

Dennis Sperlich, on behalf of the ATLAS Collaboration

Humboldt-Universität zu Berlin

\section{ATLAS inner detector upgrade}



\section{Stave and Petal}

- ITk strip detector is build from 2 multi-module assemblies - staves in the barrel

- petals in the end-caps

- provide power, cooling and data to the individual modules

- modules glued to structure

- double sided with incorporated 40-52 mrad stereo angle

$-r \phi$ resolution: $20-23 \mu \mathrm{m}$

$-z$ resolution: $850 \mu \mathrm{m}$

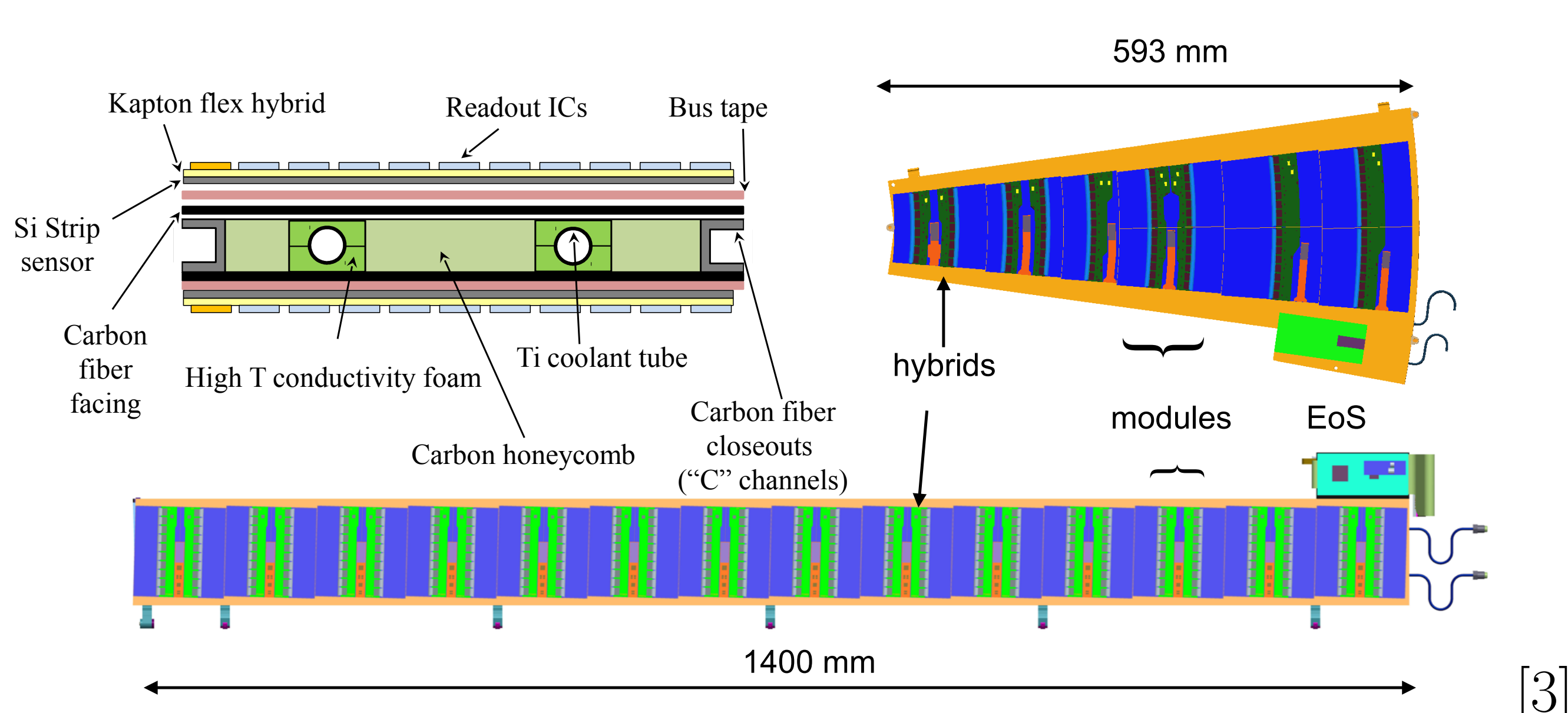

- evaporative $\mathrm{CO}_{2}$ cooling down to approximately $-35^{\circ} \mathrm{C}$ at the end of life of the experiment

- data transmission up to $2 \times 640 \mathrm{MBit} / \mathrm{s}$ per module

- conversion to $2 \times 10.24 \mathrm{GBit} / \mathrm{s}$ fiber at end of structure

- up to $2 \times 9.3 \mathrm{~A} @ 11 \mathrm{~V}$
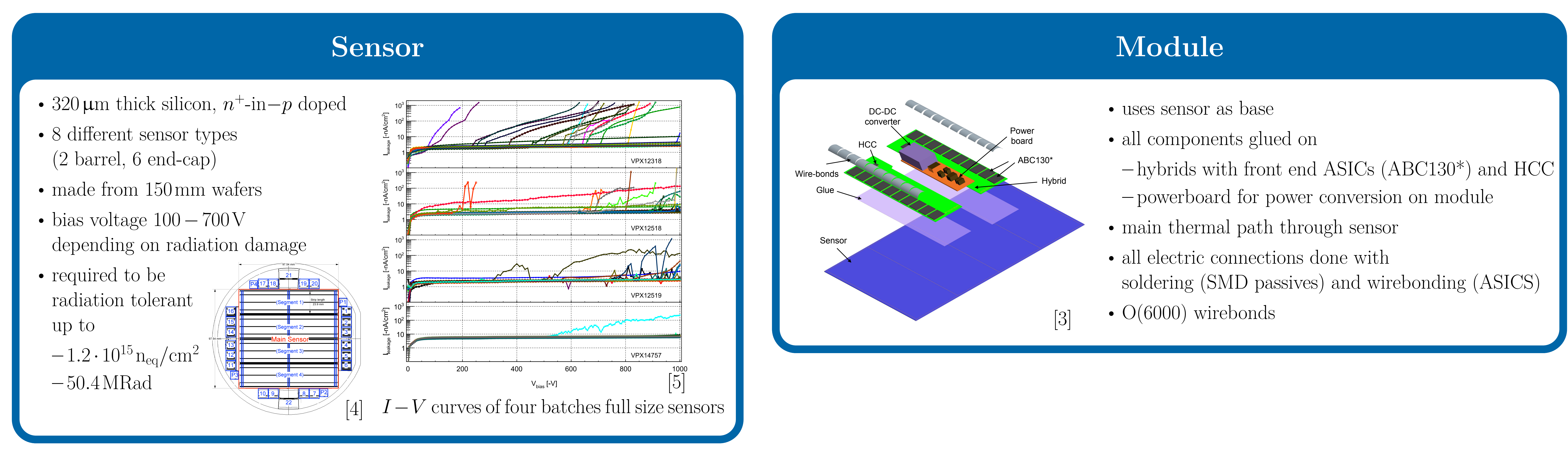

\section{Powerboard}

- generates 3 A@1.5V from $11 \mathrm{~V}$

- contains analog monitoring

- LV voltage and current

- HV current

- temperature
- radiation hard DCDC ASIC (upFEAST)

- ASIC for slow control and autonomous safety (AMAC)

- HV filter and switch

- can disconnect single modules from HV

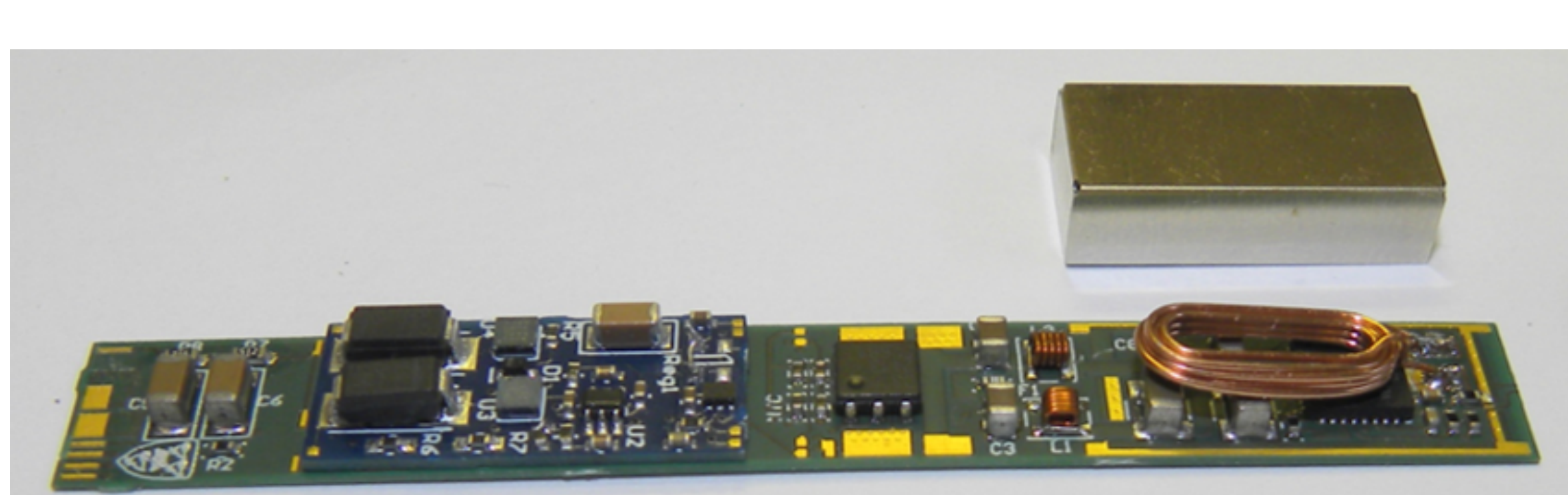

HV filter HV switch Control DCDC Power Block

\section{ATLAS Binary Chip (ABC)}

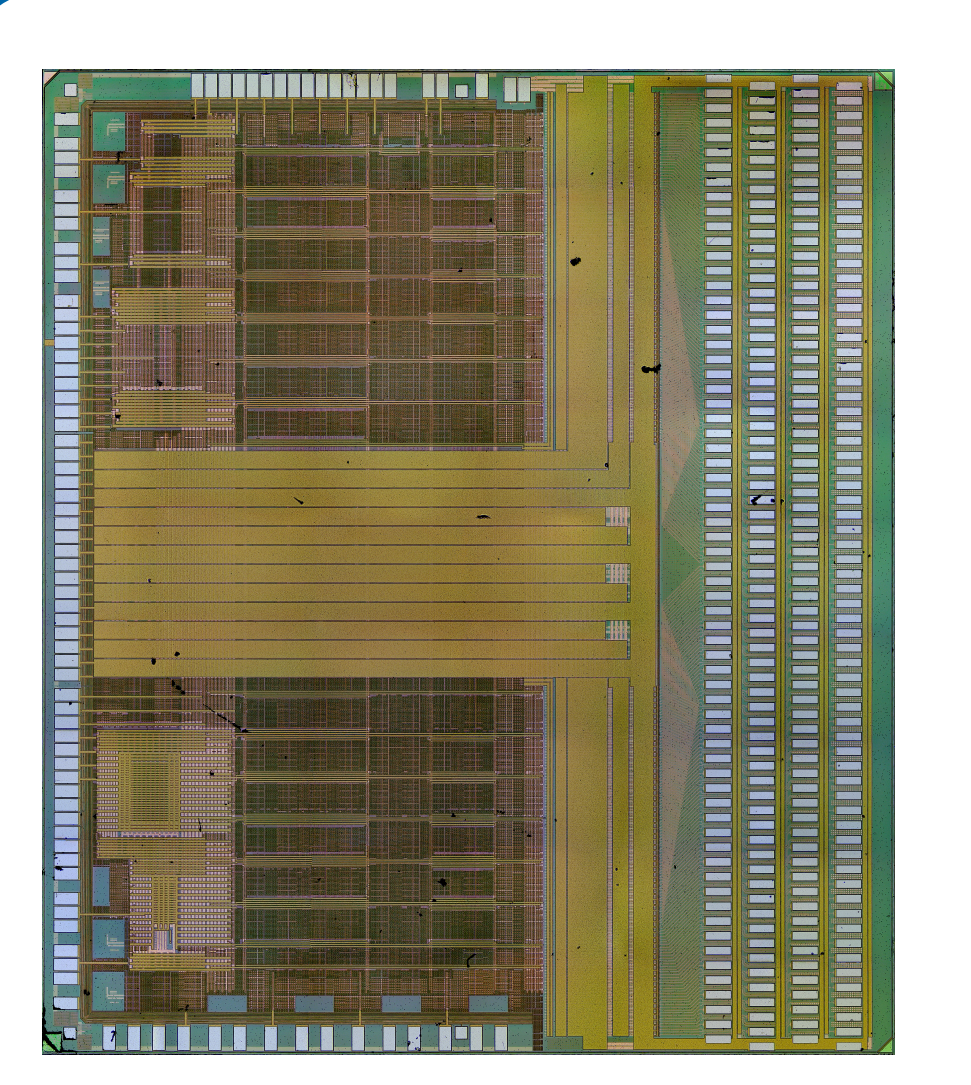

- converts analog signal from strip sensor to binary hit data

- wirebonded directly to 256 strips

- processes signals from 256 strips

- buffers for $12.8 \mu \mathrm{s}+128$ events

- matches ATLAS trigger scheme and timing

- compresses data (zero suppression) and builds packets

\section{Hybrid Control Chip (HCC)}

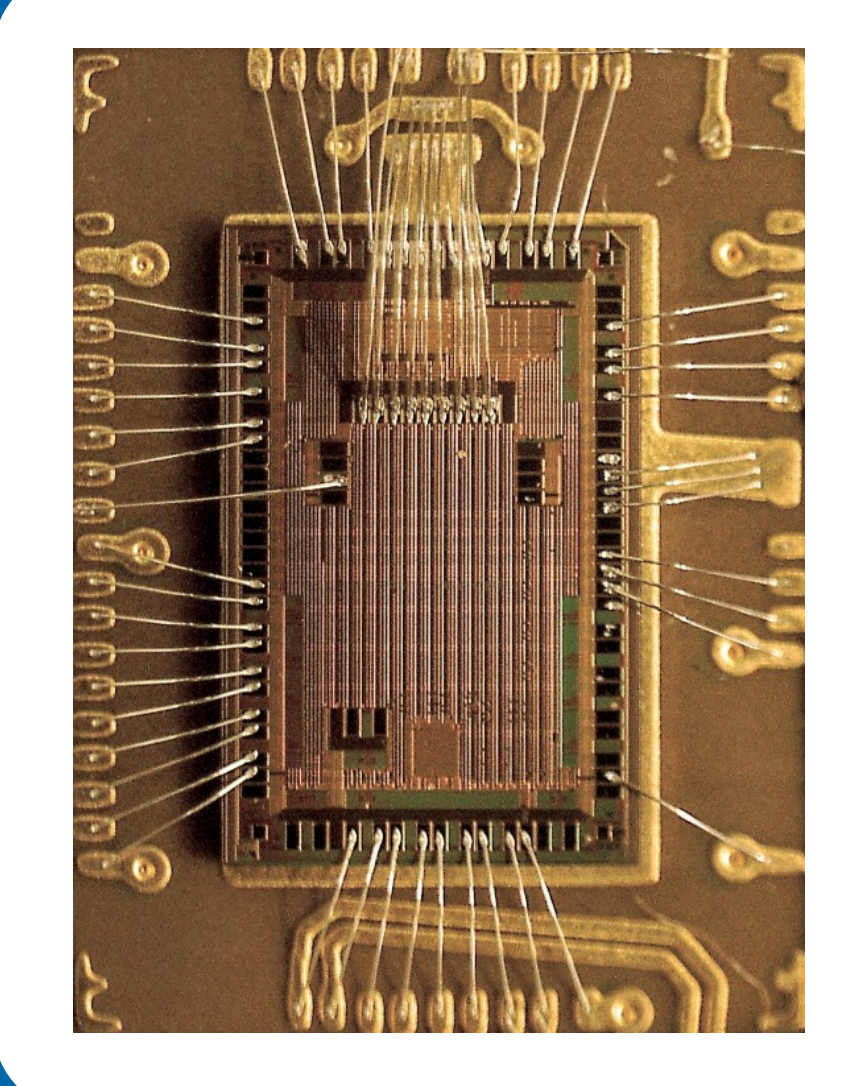

- interface between stave/petal and hybrid

- creates common bus of control signals for ABC $(2 \times 160 \mathrm{MBit} / \mathrm{s})$

- collects hit data from up to $11 \mathrm{ABC}(160 \mathrm{MBit} / \mathrm{s}$ each)

- packetize and multiplexes data from all $\mathrm{ABC}$ per event

- outgoing data to stave/petal up to $640 \mathrm{MBit} / \mathrm{s}$

- generates data clocks from bunch crossing clock

\section{References}

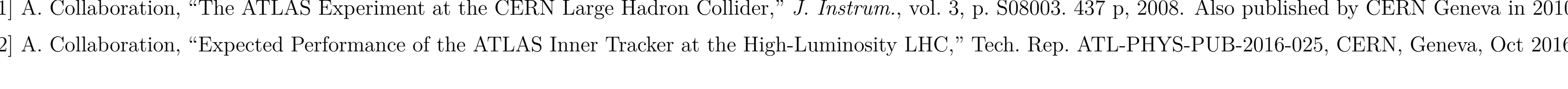

A. Collaboration, "Technical Design Report for the AILAS ITk-Strips Detector," Tech. Rep. AIL-COM-UPGRADE-2016-033, CERN, Geneva, 2016. unpublished.

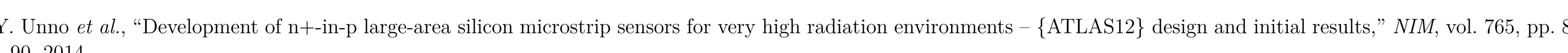
L. Hommels et all, "Detailed studies of full-size \{ATLAS12\} sensors," NIM, vol. 831, pp. 167 - 173, 2016 